\title{
Pericytes and the blood-brain barrier: recent advances and implications for the delivery of CNS therapy
}

\begin{abstract}
"Once the regulation of brain endothelial transcytosis is understood at the molecular level, it should be possible to exploit these mechanisms as targets for facilitated CNS drug delivery."
\end{abstract}

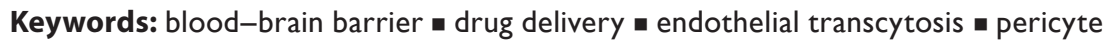

For a 'neurocentric' neurobiologist, the blood vessels in the brain often go unnoticed, for the pharmaceutical companies working on drugdelivery platforms they are a major problem. The field of vascular biology is young and has not experienced an explosion of studies dedicated to the understanding of the complexity of the brain vasculature. However, much can be learned from the intersection of the fields of neurobiology and vascular biology, with future exploration of blood vessel specification offering new drug-delivery platforms into the CNS and insight into communication between different cell types in the brain.

The majority of blood vessels consist of two cell types: endothelial cells that line the lumen of a vessel and mural cells (vascular smooth muscle cells and pericytes) that line the abluminal side of the vessels. The term 'pericyte' is applied to mural cells lining both micro- and medium-sized vessels. The CNS endothelium has the highest pericyte coverage: the length of coverage approaches $100 \%$, whereas only a third of the vessel circumference is covered by pericyte processes [1]. In addition, the entire abluminal side of CNS blood vessels is wrapped by glial processes termed astrocyte end-feet [1]. Thus, the term 'neurovascular unit' is often used to highlight complex cell-cell interactions around the brain vasculature.

\section{The neurovascular unit}

The cellular components of the neurovascular unit in the adult brain are endothelial cells, pericytes and astrocytes, but also include associated microglia and nerve endings. All cellular components except astrocytes, appear in the CNS early during embryonic development. During murine development, endothelial cells and pericytes migrate into the neuroepithelium around E9-10.5 [2]. Simultaneously, microglia precursors start to colonize the CNS [3]. The creation and specification of astrocytes occurs around birth at defined regions in the brain, while investment of blood vessels with astrocyte end-feet is completed at 2 weeks after birth in mice $[4,5]$. Taking into account the time of origin of all cellular components of the neurovascular unit, the full maturation of the neurovascular unit likely takes place after birth.

\section{The blood-brain barrier: not only endothelial junctions}

Reese and Karnovsky demonstrated in 1967 that the mammalian blood-brain barrier (BBB) is endothelium based [6]. CNS endothelial cells possess several characteristics that restrict the paracellular and transcellular passage of polar molecules and ions from blood to brain. Cell-cell contacts between endothelial cells are tightly sealed, restricting the paracellular passage of molecules. In addition, endothelial cells show a low rate of transcytosis, and they express drug efflux transporters that remove potentially toxic xenobiotics from the endothelium and parenchyma. The CNS endothelial cells have specific transport systems that allow them to supply the brain with necessary nutrients, ions and bioactive molecules. Collectively, these particular characteristics of the brain vasculature also make drug delivery into the CNS a challenge.

\section{Development \& specification of the brain vasculature}

The CNS is vascularized by sprouting angiogenesis, a process in which the formation of new blood vessels occurs by induction of migratory endothelial tip cells in pre-existing vessels. These

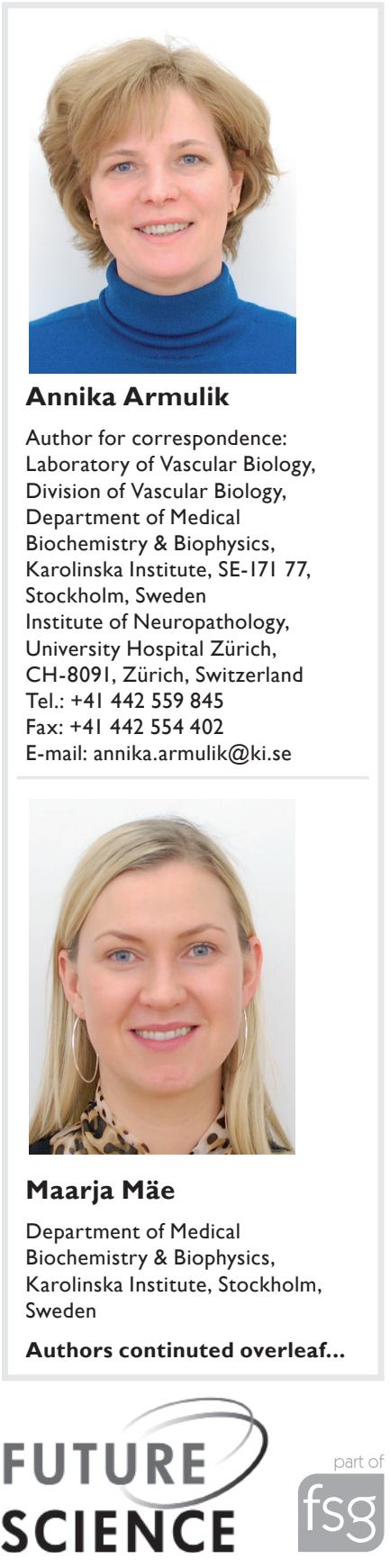




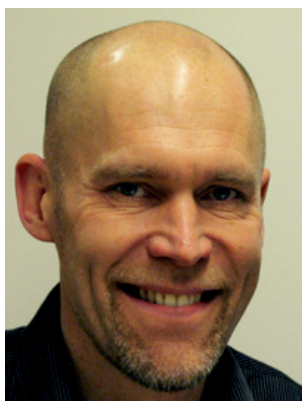

Christer Betsholtz

Department of Medical Biochemistry \& Biophysics, Karolinska Institute, Stockholm, Sweden tip cells then spearhead and guide elongating vascular sprouts into the CNS [7]. The sprouts emanate from a perineural vascular plexus of mesodermal origin that forms around the developing neural tube at an earlier developmental stage [8]. CNS pericytes, derived from the cranial neural crest, ingress into the developing neural tissue together with endothelial cells [9]. Undoubtedly, the vessels entering the CNS during the development do not possess all the characteristics of adult brain endothelial cells. However, at this stage of development, endothelial cells already form well-sealed cell-cell junctions that restrict the passage of plasma proteins into the CNS [10].

"...much can be learned from the intersection of the fields of neurobiology and vascular biology, with future exploration of blood vessel specification offering new drug delivery platforms into the CNS..."

Transplantation studies in embryos have demonstrated that signals inducing the formation of tight endothelial junctions are intrinsic to the neuronal tissue [11], but the molecular nature of the inducing signal has remained elusive. Recent studies have demonstrated that the neuroepithelium secretes Wnt7a/7b, which binds to a yet unidentified receptor on endothelial cells to induce canonical Wnt/ $\beta$-catenin signaling [12]. This leads to the formation of tightly sealed endothelial junctions and expression of the brain endothelial cell-specific glucose transporter (GLUT)-1. Whereas these BBB characteristics develop as soon as the blood vessels enter into the neuroepithelium, other BBB characteristics develop at a later time. It is known that the expression of the ATP-binding cassette (ABC) transporters (e.g., P-glycoprotein) in brain vasculature starts after birth. Regulation of $A B C$ transporters is complex, and the expression of these transporters depends on age and on the metabolic/disease states of the organism [13].

Reese and Karnovsky demonstrated that in addition to the sealed interendothelial junctions that restrict the passage of the exogenous tracer horseradish peroxidase, the brain endothelial cells show a low vesicular uptake of horseradish peroxidase that is not transported to the abluminal side [6]. Indeed, electron microscopy studies have demonstrated low-vesicular content of brain endothelial cells [14]. However, this does not exclude the possibility that brain endothelial cells are equipped with the machinery for transcytosis. The brain vasculature facilitates the entry of many proteins into the brain by receptor-mediated transcytosis (e.g., transferrin), a feature that has been used to design delivery platforms for pharmaceuticals into the brain [15]. The molecular mechanism(s) by which brain endothelial cells maintain a low rate of vesicular transport is not known. However, two recent studies by Daneman et al. and our group have demonstrated that pericytes regulate endothelial transcytosis at the BBB in vivo $[16,17]$.

\section{Pericytes \& the regulation of the BBB/neurovascular unit}

It is well established that adequate pericyte coverage is important for the formation of durable vessels with appropriate lumen diameter during embryonic development. Our current knowledge about the role(s) of pericytes in the adult organism is still fragmentary. The view that pericytes are important regulators of the BBB has long been propagated, although it was based on only a few experimental studies performed in cell culture. These studies suggested that pericytes regulate endothelial junctions and thus, they might be involved in regulating the BBB in vivo $[18,19]$.

The lack of viable pericyte-deficient animal models, as well as methods for the unambiguous identification of pericytes, have hampered studies aimed at addressing the role of pericytes in adult organisms. In order to promote the analysis of pericyte functions in the adult organism, we have developed a series of mouse models that are adult viable, which have greatly reduced pericyte coverage of the CNS vasculature, by manipulating the PDGF-B/PDGF receptor (PDGFR)- $\beta$ signaling axis $[17,20]$. Analysis of these adult pericyte-deficient mice showed that the degree of pericyte deficiency correlated with the relative permeability of brain vasculature to a variety of tracers [17]. Similar conclusions were drawn by Daneman et al. in their analysis of an allelic series of PDGFR- $\beta$ hypomorphs with various degrees of pericyte coverage [16].

Analysis of $p d g f r b^{-1-}$ embryos demonstrated that pericytes already regulate the endothelial permeability during embryogenesis [16]. Interestingly, whereas earlier in vitro studies had suggested that pericytes might regulate vascular permeability by opening or closing the endothelial junctions, this was not the case in vivo. While endothelial junctions are broader and more convoluted in the absence of pericytes, they express junctional markers normally. Moreover, 
these junctions are continuous and do not show accumulation of intravenously injected tracers $[16,17]$. Instead, the experimental data points to increased vesicular transport in the endothelium as a cellular mechanism for the increased permeability. Intravenously administered horseradish peroxidase could be detected in large vesicles in the endothelium of pericyte-deficient mice, as well as at the endothelial basement membrane, indicating a transcellular route of passage $[16,17]$. Currently, it is unclear which type(s) of vesicular uptake in the endothelium is misregulated in the absence of pericytes. However, the presence of large vesicles $(>200 \mathrm{~nm})$ is an indication of macropinocytosis.

In $p d g f r b^{-1-}$ embryos the brain vessels showed ectopic expression of Plvap, a protein associated with endothelial fenestrae [16]. In adult pericytedeficient mice the expression of Plvap could not be detected [17]. This discrepancy could reflect a difference in the degree of pericyte loss, or, more likely, that adult mice possess alternative mechanisms to suppress Plvap expression.

Interestingly, administration of the tyrosine kinase inhibitor imatinib blocked the extravasation of intravenously injected tracers into the brain parenchyma. However, imatinib treatment led to vesicular accumulation of the tracer in the endothelium. This indicates that the molecular target of imatinib is involved in regulating exocytosis of the tracer-containing vesicles. Therefore, pericytes, which inhibit the endocytotic uptake of tracer-containing vesicles into endothelial cells, and imatinib, which inhibits their release, appear to work through different mechanisms to inhibit transcytosis.

\section{"...the specific question of how vesicular transport across differentiated brain endothelial cells is regulated is too complicated to address using current in vitro models. In vivo analysis may be a more fruitful approach..."}

Besides the misregulated vesicular transport, are there other characteristics of brain-specific endothelium that are affected in pericytedeficient mice? Several aspects of brain endothelial cell characteristics do not seem to be affected by the absence of pericytes. Pericytes do not appear to be necessary for the induction of expression of a majority of the BBB-specific endothelial genes $[16,17]$. As previously mentioned, basic aspects of the BBB, and some brain endothelial-specific gene expression is evoked by Wnt signaling. However, the absence of pericytes leads to some specific changes in the brain endothelial gene transcription profile. The pericyte-deficient brain microvessels have downregulated transferrin receptors, both on the mRNA and protein level [17]. In addition, the upregulation of leukocyte cell adhesion molecules is observed in pericyte-deficient brain endothelium in embryos [16]. The upregulation of these adhesion molecules and an increased attachment of leukocytes to the endothelium are also seen in pericyte-deficient adults [Armulik A, Mäe M, Betsholtz C, Unpublished Data]. We could not detect leukocytes in the brain parenchyma [Armulik A, Mäe M, Betsholtz C, Unpublished Data], indicating that even if there is an increased attachment, these cells do not cross the endothelium. Importantly, in the absence of pericyte coverage, the brain endothelial cells show correct apical-basal polarization [17]. It has been suggested that pericytes regulate the assembly of the endothelial basement membrane but pericyte-deficient brain vessels did not show any dramatic changes in deposition of major components of the basement membrane [17]. We noticed, however, a strong reduction of astrocytederived laminin $\alpha 2$ chain in the basement membrane. This observation prompted us to analyze the astrocyte end-feet in pericyte-deficient vessels. Localization of several astrocyte end-feet markers showed a fuzzy staining not associated with vessels, indicating a defective polarization of astrocyte end-feet.

Thus, recent in vivo analysis of pericytedeficient mouse models demonstrates a role for pericytes in regulating endothelial permeability at the level of transcytosis. Unraveling the molecular mechanisms of this rather unexpected route of transport across the BBB could open a new paradigm for the development of CNS drug-delivery strategies.

\section{Conclusion \& future perspective}

Our knowledge about the regulation of brain endothelial transcytosis is scant; clearly, more basic research is required in this area. Once the regulation of brain endothelial transcytosis is understood at the molecular level, it should be possible to exploit these mechanisms as targets for facilitated CNS drug delivery. Most likely, endothelial cells will turn out to use many of the same mechanisms for vesicular transport as other eukaryotic cells, such as the use of cortical actin filaments for vesicle generation, Rab GTPases for cargo targeting/transport, and SNARE complexes for exocytosis. The BBB is, to a major extent, a core characteristic of brain 
endothelial cells that is induced/maintained/ regulated by the environment in which the brain endothelial cells reside, and no available cell culture model appears to recapitulate such an environment. Thus, the specific question of how vesicular transport across differentiated brain endothelial cells is regulated is too complicated to address using current in vitro models. In vivo analysis may be a more fruitful approach, at least until improved experimental setups, representing the complexity of the neurovascular unit, become available. Pericyte-deficient mice may henceforth serve as valuable tools for discovery, since pericytes are key (down)regulators of brain endothelial transcytosis $[16,17]$. In vivo approaches will not be suitable for high-throughput drug screening, nor for detailed biochemical analysis of signaling pathways, but they may be valuable for proof-of-principle experiments and for unbiased analysis of brain microvessel transcriptomes and proteomes in the transcytosis-activated and inactivated states.

\section{Financial \& competing interests disclosure}

The authors have no relevant affiliations or financial involvement with any organization or entity with a financial interest in or financial conflict with the subject matter or materials discussed in the manuscript. This includes employment, consultancies, honoraria, stock ownership or options, expert testimony, grants or patents received or pending, or royalties.

No writing assistance was utilized in the production of this manuscript.

\section{Bibliography}

1 Mathiisen TM, Lehre KP, Danbolt NC, Ottersen OP. The perivascular astroglial sheath provides a complete covering of the brain microvessels: an electron microscopic 3D reconstruction. Glia 58(9), 1094-1103 (2010).

2 Vasudevan A, Long JE, Crandall JE, Rubenstein JL, Bhide PG. Compartmentspecific transcription factors orchestrate angiogenesis gradients in the embryonic brain. Nat. Neurosci. 11(4), 429-439 (2008).

3 Ransohoff RM, Cardona AE. The myeloid cells of the central nervous system parenchyma. Nature 468(7321), 253-262 (2010).

4 Rowitch DH, Kriegstein AR. Developmental genetics of vertebrate glial-cell specification. Nature 468(7321), 214-222 (2010).

5 Landis DM, Reese TS. Membrane structure in mammalian astrocytes: a review of freeze-fracture studies on adult, developing, reactive and cultured astrocytes. J. Exp. Biol. 95, 35-48 (1981).

6 Reese TS, Karnovsky MJ. Fine structural localization of a blood-brain barrier to exogenous peroxidase. J. Cell Biol. 34(1), 207-217 (1967).

7 Gerhardt H, Golding M, Fruttiger M et al. VEGF guides angiogenic sprouting utilizing endothelial tip cell filopodia. J. Cell. Biol. 161(6), 1163-1177 (2003).
8 Hogan KA, Bautch VL. Blood vessel patterning at the embryonic midline. Curr. Top. Dev. Biol. 62, 55-85 (2004).

9 Etchevers HC, Vincent C, Le Douarin NM, Couly GF. The cephalic neural crest provides pericytes and smooth muscle cells to all blood vessels of the face and forebrain. Development 128(7), 1059-1068 (2001).

10 Saunders NR, Habgood MD, Dziegielewska KM. Barrier mechanisms in the brain, II. Immature brain. Clin. Exp. Pharmacol. Physiol. 26(2), 85-91 (1999).

11 Stewart PA, Wiley MJ. Developing nervous tissue induces formation of blood-brain barrier characteristics in invading endothelial cells: a study using quail-chick transplantation chimeras. Dev. Biol. 84(1), 183-192 (1981).

12 Liebner S, Plate KH. Differentiation of the brain vasculature: the answer came blowing by the Wnt. J. Angiogenes. Res. 2 DOI:10.1186/2040-2384-2-1 (2010) (Epub ahead of print).

13 Miller DS. Regulation of P-glycoprotein and other ABC drug transporters at the bloodbrain barrier. Trends Pharmacol. Sci. 31(6), 246-254 (2010).

14 Coomber BL, Stewart PA. Three-dimensional reconstruction of vesicles in endothelium of blood-brain barrier versus highly permeable microvessels. Anat. Rec. 215(3), 256-261 (1986).
15 Pardridge WM. Biopharmaceutical drug targeting to the brain. J. Drug Target 18(3), 157-167 (2010).

16 Daneman R, Zhou L, Kebede AA, Barres BA. Pericytes are required for blood-brain barrier integrity during embryogenesis. Nature 468(7323), 562-566 (2010).

17 Armulik A, Genove G, Mae M et al. Pericytes regulate the blood-brain barrier. Nature 468(7323), 557-561 (2010).

18 Hori S, Ohtsuki S, Hosoya K, Nakashima E, Terasaki T. A pericyte-derived angiopoietin-1 multimeric complex induces occludin gene expression in brain capillary endothelial cells through Tie-2 activation in vitro. J. Neurochem. 89(2), 503-513 (2004).

19 Dohgu S, Takata F, Yamauchi A et al. Brain pericytes contribute to the induction and up-regulation of blood-brain barrier functions through transforming growth factor- $\beta$ production. Brain Res. 1038(2), 208-215 (2005).

20 Lindblom P, Gerhardt H, Liebner S et al. Endothelial PDGF-B retention is required for proper investment of pericytes in the microvessel wall. Genes Dev. 17(15), 1835-1840 (2003). 